PHYSICAL REVIEW D 95, 029902(E) (2017)

\title{
Erratum: Spin precession of slow neutrons in Einstein-Cartan gravity with torsion, chameleon, and magnetic field \\ [Phys. Rev. D 93, 045031 (2016)]
}

A. N. Ivanov and M. Wellenzohn

(Received 22 December 2016; published 9 January 2017)

DOI: 10.1103/PhysRevD.95.029902

We add the following sentence to the Acknowledgments:

This work was also supported by the Austrian Fonds zur Förderung der Wissenschaftlichen Forschung (FWF) under Contracts No. I862-N20 and No. P26781-N20. 